\title{
Acquisition and extinction of context-specific morphine withdrawal
}

\author{
CHERYL DEFFNER-RAPPOLD, JULIAN L. AZORLOSA, and JEFFREY D. BAKER \\ Southeastern Louisiana University, Hammond, Louisiana
}

\begin{abstract}
The acquisition, retention, and extinction of context-specific morphine withdrawal in rats were investigated in two experiments. In Experiment 1, context-specific withdrawal was observed after a placebo injection following 11 sessions in which the context was paired with either a $10-\mathrm{mg} / \mathrm{kg}$ or a $75-\mathrm{mg} / \mathrm{kg}$ dose of morphine. Contextual withdrawal was retained during 10 days of drug abstinence in both dose conditions. In Experiment 2, context-specific withdrawal (rearing) was retained after 21 days of morphine abstinence. In addition, conditioned rearing was extinguished by exposure to the drug context with or without a placebo injection. Injection cues did not contribute to conditioned withdrawal.
\end{abstract}

Chronic administration of morphine and other opiates produces both tolerance and dependence. Tolerance is defined as a decrease in drug effectiveness that occurs after repeated administration, and dependence is identified by the appearance of withdrawal symptoms after the drug is abruptly discontinued. A large body of research has shown that tolerance to many of the effects of morphine (e.g., analgesia, hyperthermia, sedation) is at least partly mediated by environmental or contextual cues paired with the drug (for a review, see Goudie \& Demellweek, 1986).

Siegel $(1975,1989)$ proposed a classical conditioning model of drug tolerance in which contextual cues present during drug administration serve as a conditioned stimulus (CS) and the drug acts as the unconditioned stimulus (US). After repeated pairings of these cues and the drug, the cues elicit compensatory, drug-opposite responses. These compensatory conditioned responses (CRs) serve a homeostatic function and reduce the drug's effects, which results in tolerance.

Siegel's model $(1975,1989)$ also incorporates drug dependence. In the absence of drug administration, the environmental cues still elicit the compensatory CRs, which are withdrawal signs. Tolerance and dependence, according to this model, result from the same underlying process. Although contextual control of tolerance to opiates is well documented (see Goudie \& Demellweek, 1986; Siegel, 1989), its mediation by compensatory CRs is controversial. Siegel and others have found cue-elicited compensatory hyperalgesia (Siegel, 1975), hypothermia (Siegel, 1978), and hyperactivity (Hinson \& Siegel, 1983; Mucha, Volkovskis, \& Kalant, 1981). However, others have offered alternative explanations for these demonstrations

This research was submitted by the first author in partial fulfillment of the requirements for the master's degree in psychology at Southeastern Louisiana University. The morphine sulfate was a generous gift from the National Institute on Drug Abuse. Correspondence concerning this article should be addressed to $\mathbf{J}$. L. Azorlosa, SLU 324, Hammond, LA 70402 (e-mail: jazorlosa@selu.edu). or have failed to find evidence of compensatory responding in tolerant subjects (see Goudie \& Demellweek, 1986).

In spite of the controversy about cue-elicited compensatory responses, there is evidence that cues repeatedly paired with morphine can elicit opiate withdrawal signs such as rearing, circling, jumping, darting, genital licking, and wet dog shakes (Azorlosa, Hartley, \& DeffnerRappold, 1994; Falls \& Kelsey, 1989; Kelsey, Aranow, \& Matthews, 1990; Krank \& Perkins, 1993; but see Sobrero \& Bouton, 1989). It has also been shown that contextual withdrawal is retained for at least a week of drug abstinence (Azorlosa et al., 1994). Retention of contextual withdrawal is important, because these cues may trigger relapse after a long period of drug abstinence (Hinson, Poulos, Thomas, \& Cappell, 1986).

The first experiment was conducted to determine whether cues repeatedly paired with different doses of morphine would elicit different withdrawal signs and whether contextual withdrawal would be retained for a longer time than had previously been reported. The second experiment was conducted to determine whether context-specific withdrawal could be extinguished.

In Experiment 1, we investigated the effects of two different morphine doses on the acquisition and retention of contextual withdrawal. In other, previous studies, doses had been administered in an ascending series, terminating at $50 \mathrm{mg} / \mathrm{kg}$ (Falls \& Kelsey, 1989) or $40 \mathrm{mg} / \mathrm{kg}$ (Kelsey et al., 1990; Krank \& Perkins, 1993). In the present study, we used lower $(10-\mathrm{mg} / \mathrm{kg})$ and higher $(75-\mathrm{mg} / \mathrm{kg})$ terminal doses. We had already found context-specific withdrawal with a $10-\mathrm{mg} / \mathrm{kg}$ dose (Azorlosa et al., 1994), but there have been no demonstrations of contextual withdrawal with a dose as high as $75 \mathrm{mg} / \mathrm{kg}$ and none in which the effects of two different doses have been compared. Such a comparison is important, because studies of unconditioned morphine withdrawal have shown that the appearance and intensity of various signs vary according to dose (e.g., Blasig, Herz, Reinhold, \& Zieglgänsberger, 1973). 


\section{EXPERIMENT 1}

\section{Method}

Subjects. Subjects were 40 male Sprague-Dawley rats weighing $218-279 \mathrm{~g}$ at the start of the experiment. These animals were individually housed in the colony room and were allowed ad-lib access to food and water. The colony room was lighted on a 14:10-h light:dark cycle. The animals were run during the lighted part of the cycle.

Drugs. Morphine sulfate was administered in a $1-\mathrm{ml} / \mathrm{kg}$ vehicle of $.85 \%$ sodium chloride. All injections were administered subcutaneously in the dorsal neck area.

Distinctive environment. The distinctive environment (DE) consisted of two rooms, each containing four aluminum cages with black and white stripes on the sides and floor. White noise masked extraneous noise and contributed to the distinctiveness of the DE. A partially covered lamp with a $45-\mathrm{W}$ bulb illuminated Room 1. In Room 2, desk lamps with $25-\mathrm{W}$ red bulbs were mounted above each cage. The cages were cleaned with a pinescented disinfectant between sessions.

Procedure. On the first 3 days, the rats were habituated to handling and injections. On the 1st day, they were weighed and injected with saline. Thirty minutes later, they received a second saline injection. This procedure was repeated on the next 2 days.

The day after pretraining, the animals were randomly assigned to one of five groups and given 11 acquisition sessions in the DE. The sessions lasted $1 \mathrm{~h}$ and were spaced $48 \mathrm{~h}$ apart. This interval ensured that there were no residual drug effects from the previous session. The two paired groups $(\mathrm{P})$ received morphine in the $\mathrm{DE}$ and saline in the home cage (HC). The two unpaired groups (U) received saline in the DE and morphine in the HC. P75 and U75 received an ascending dose of morphine $(5,10,20,30,30,50,50$, $75,75,75,75 \mathrm{mg} / \mathrm{kg}$ ). Groups $P 10$ and U10 received an initial dose of $5 \mathrm{mg} / \mathrm{kg}$ followed by $10 \mathrm{mg} / \mathrm{kg}$ for all remaining sessions. Group Sal received saline in both the DE and HC. During each acquisition trial, the animals were carried to the assigned room in the $\mathrm{DE}$, injected with the appropriate drug, and placed in the assigned cage, where they remained undisturbed for $60 \mathrm{~min}$. Room and cage assignment were counterbalanced among groups. Home cage injections were given $5 \mathrm{~h}$ after each session. The animals were removed from the cage, injected, and returned to the cage. Twenty-four hours after the last acquisition trial, withdrawal was tested. The animals were carried to the DE and injected with saline. For $10 \mathrm{~min}$ immediately after the injection, the following withdrawal signs were recorded: rears (number of times animal stood on its hind paws, stretching its torso upward), wet dog shakes (brief shakes of head and body), jumps (all four paws off the ground), circles (continuous rotation), and darts (swift movements from one area of the cage to another). These signs have been used as indices in both pharmacological studies of morphine withdrawal (e.g., Blasig et al., 1973; Linesman, 1977) and studies of conditioned withdrawal (Azorlosa et al., 1994; Falls \& Kelsey, 1989; Kelsey et al., 1990; Krank \& Perkins, 1993). Twenty-four hours after the first withdrawal test, the groups received one additional acquisition trial to compensate for any effects of the test. The animals then remained undisturbed in their home cages for 9 days. On the 10th day, the rats were tested for retention of withdrawal.

There were two observers who were blind to the animals' group assignment. Prior to withdrawal testing, interobserver reliability was measured $(r=.98)$.

\section{Results}

Two rats died during the experiment, 1 P75 and 1 U75 animal, leaving these groups with 7 animals.

Figure 1 shows the mean number of rears observed during the first withdrawal test conducted $24 \mathrm{~h}$ after the final morphine session. In each dose condition, the paired

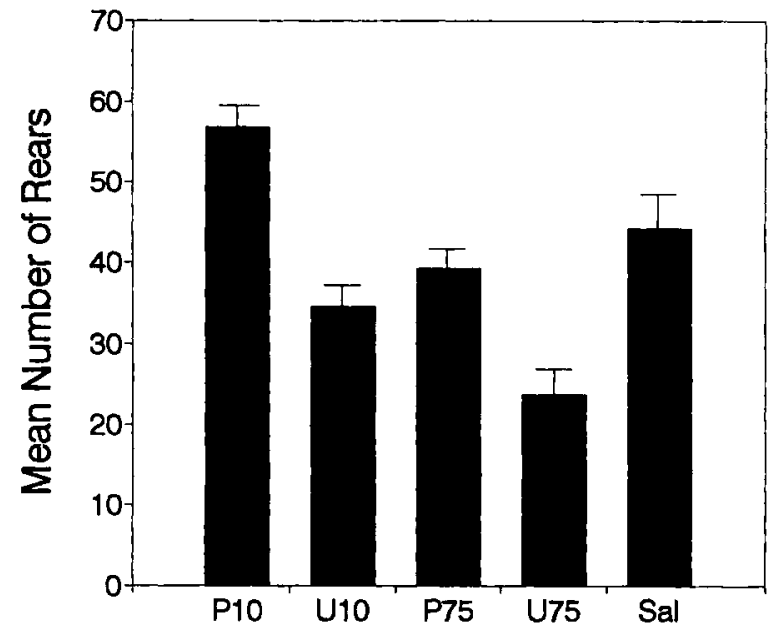

Figure 1. Mean rearing scores during the first withdrawal test in Experiment 1. Bars indicate standard error of the mean.

group reared more than the unpaired group, and the 10 $\mathrm{mg}$ dose produced more rearing than did the $75-\mathrm{mg}$ dose. A one-way analysis of variance (ANOVA) indicated a significant main effect for groups $[F(4,33)=11.98, p<$ $.001]$. Newman-Keuls comparisons $(p<.05)$ showed that P10 reared more than U10 and Sal. P75 reared more than U75 but did not differ from Sal. P10 animals also exhibited more rears than did P75 and U75.

Figure 2 presents the mean number of wet dog shakes and suggests that this sign was context-specific only in the high-dose condition. A one-way ANOVA revealed a significant effect for groups $[F(4,33)=3.84, p<.01]$. Newman-Keuls comparisons showed that P75 animals exhibited significantly more wet dog shakes than did both U75 and Sal. There were no other reliable differences. One-way ANOVAs revealed no significant effects for darts, jumps, or circles (not shown).

Mean number of rears observed during the withdrawal retention test are displayed in Figure 3. A one-way ANOVA

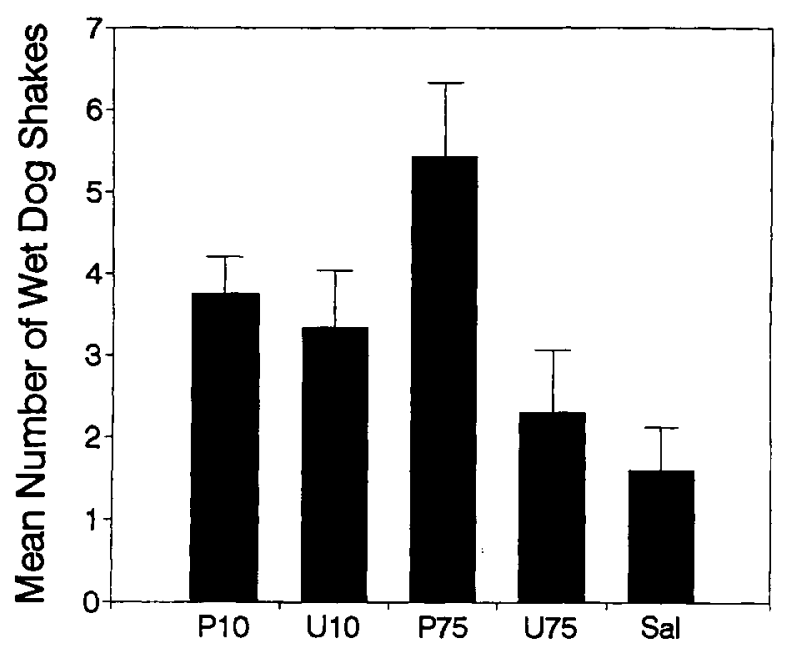

Figure 2. Mean wet dog shakes during the first withdrawal test in Experiment 1. 
revealed a significant effect for groups $[F(4,33)=9.35$, $p<.001]$. Newman-Keuls comparisons showed that $\mathrm{P} 75$ reared more than $\mathrm{U} 75$ and Sal and that $\mathrm{P} 10$ reared more than both U10 and Sal. Neither unpaired group differed from Sal. One-way ANOVAs revealed no significant effects for the other signs.

Although the ANOVAs showed no significant effects for the other withdrawal signs, the analyses of darts, jumps, and circles showed marked heterogeneity of variances for the scores for all three signs. Therefore, a nonparametric test, the Mann-Whitney $U$ (Siegel, 1956) was used to test rank order effects $(p<.05)$. These tests, and the median scores of darts, circles, and jumps, are summarized in Table 1. During the original withdrawal test, P10 displayed more darts and jumps than did P75. Both P75 and U75 exhibited fewer darts, jumps, and circles than did Group Sal. A very different pattern was evident on the retention test. P10 jumped more than both U10 and Sal. P75 jumped more than U75, but did not differ from Sal. Similarly, P10 darted more than U10, but the difference between P10 and Sal only approached significance $(p=.06)$.

\section{Discussion}

Experiment 1 showed that contextual cues previously paired with morphine elicited withdrawal and that contextspecific withdrawal was retained during a 10 -day period of drug abstinence. This experiment also showed that different doses produced a different pattern of contextual withdrawal $24 \mathrm{~h}$ after the final morphine injection. For example, both $\mathrm{P} 10$ and $\mathrm{P} 75$ reared more than their respective unpaired controls, but P75 did not differ from Sal, which makes contextual control of rearing in the high dose problematic. Wet dog shakes were controlled by the context only with the higher $(75-\mathrm{mg} / \mathrm{kg})$ dose. In addition, the overall level of activity in Groups P75 and U75 was lower than in $\mathrm{P} 10$ and $\mathrm{U} 10$, respectively. P75 and U75 reared less than P10 and U10, respectively (see Fig-

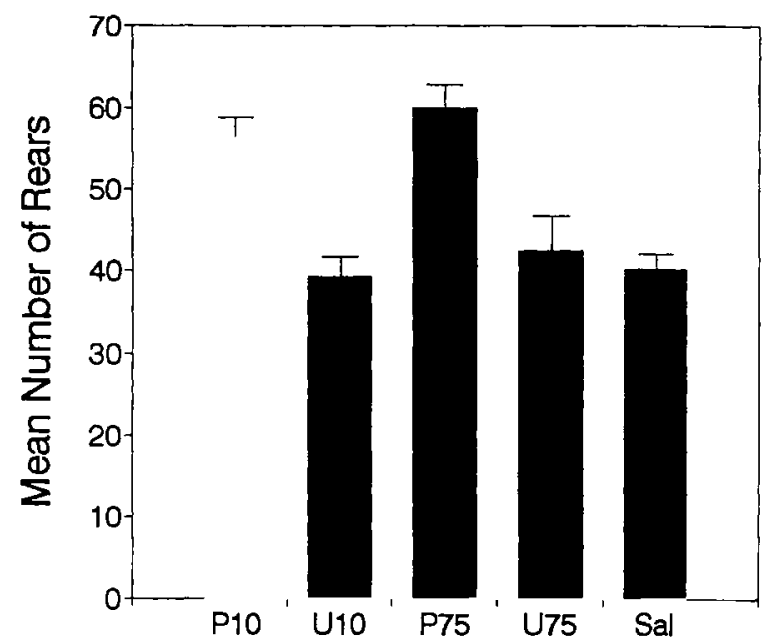

Figure 3. Mean rearing scores during the withdrawal retention test in Experiment 1.
Table 1

Median Withdrawal Scores and Mann-Whitney $U$ Tests (One-Tailed, $p<.05$ ) From Experiment 1

\begin{tabular}{llllll}
$\begin{array}{l}\text { Withdrawal } \\
\text { Sign }\end{array}$ & P75 & U75 & P10 & U10 & Sal \\
\hline \multicolumn{7}{c}{ First } & Withdrawal & Test \\
Circles & 0.0 & 0.0 & 1.0 & 0.5 & 3.5 \\
Jumps & 1.0 & 0.0 & 8.5 & 6.5 & 9.0 \\
Darts & 0.0 & 0.0 & 2.5 & 2.0 & 1.5
\end{tabular}

Circles: $\mathrm{S}>\mathrm{P} 75,(U=9) ; \mathrm{S}>\mathrm{U} 75,(U=10)$

Jumps: $\mathrm{P} 10>\mathrm{P} 75,(U=13)$; $\mathrm{Sal}>\mathrm{P} 75,(U=13)$; $\mathrm{Sal}>\mathrm{U} 75,(U=6)$

Darts: $\mathrm{P} 10>\mathrm{P} 75,(U=8)$; $\mathrm{Sal}>\mathrm{P} 75,(U=10.5)$;

$$
\mathrm{Sal}>\mathrm{U} 75,(U=9.5)
$$

Withdrawal Retention Test

$\begin{array}{llllll}\text { Circles } & 0.0 & 0.0 & 0.0 & 0.0 & 3.5 \\ \text { Jumps } & 3.0 & 0.0 & 6.0 & 1.0 & 2.0 \\ \text { Darts } & 1.0 & 0.0 & 3.5 & 0.0 & 0.5\end{array}$

Circles: Sal $>$ P75, $(U=9)$

Jumps: $\mathrm{P} 10>\mathrm{U} 10,(U=14) ; \mathrm{P} 10>\mathrm{Sal},(U=14.5)$; P75 $>$ U75, $(U=11)$

Darts: $\mathrm{P} 10>\mathrm{U10},(U=8) ; \mathrm{P} 10>\mathrm{Sal},(U=16.5, p=.06)$

Note-P, paired; U, unpaired; Sal, saline. All significant rank differences are presented; ">" indicates higher rank.

ure 1), and both P75 and U75 exhibited less jumping, circling, and darting than did the saline controls (Table 1).

The pattern of contextual withdrawal in the two dose conditions was more similar on the retention test. Contextelicited rearing and jumping were found in the $10-\mathrm{mg} / \mathrm{kg}$ dose condition and rearing was found in the $75-\mathrm{mg} / \mathrm{kg}$ condition. In addition, P10 darted more than U10, and P75 jumped more than U75. On both tests, the scores of the unpaired groups never exceeded the scores of the saline groups for any sign; all of the withdrawal observed was contextually mediated.

A puzzling aspect of these data is that the signs observed on the original and retention tests were not always the same. For example, on the original test, only rearing was seen in the $10-\mathrm{mg} / \mathrm{kg}$ condition, but rearing and jumping were observed during the retention test. In the $75-\mathrm{mg} / \mathrm{kg}$ condition, wet dog shakes were seen in the first test but only rearing was contextually controlled in retention. Although not different from the saline controls, P10 also darted more than U10, and P75 jumped more than U75. One explanation for this difference is that residual morphine reduced the level of activity on the first test, since the test occurred only $24 \mathrm{~h}$ after the previous morphine conditioning session.

A second explanation is that the first withdrawal test produced conditioning in the paired groups. Other studies have shown that withdrawal can be conditioned to cues paired with either naloxone-precipitated withdrawal (e.g., Schnur, 1992; Schnur \& Wainwright, 1992) or abstinent withdrawal (Wikler \& Pescor, 1967). In the present experiment, perhaps the withdrawal elicited by the $\mathrm{DE}$ in the paired groups during the first test resulted in withdrawal conditioning rather than extinction. This would be analogous to the fear "incubation" hypothesis 
(Eysenck, 1979), in which a CS previously paired with an aversive US is briefly presented without the US. Instead of producing extinction, this brief presentation of the CS produces more fear than do no CS-only exposures between the original CS-US training and testing of the CS (Rohrbaugh \& Riccio, 1970; Rohrbaugh, Riccio, \& Arthur, 1972). This explanation of the present results seems unlikely, for several reasons. First, incubation has only been demonstrated in fear conditioning; as far as we can determine, it has not been demonstrated in any other conditioning preparation. Even in aversive conditioning, incubation is not a well-established phenomenon. There have been several failures to obtain it (Chorot \& Sandin, 1993; Kaloupek, 1983; Nicholaichuk, Quesnel, \& Tait, 1982), and alternative interpretations have been offered for the studies in which it was purportedly demonstrated (see the open peer commentary by McAllister and McAllister in Eysenck, 1979). Second, after the initial test in Experiment 1, the rats were given an additional acquisition trial, which may have produced more conditioning and which therefore could account for the higher number of hyperactivity signs seen in retention. However, if additional conditioning occurred on that trial, it makes the lack of retention of wet dog shakes in P75 more difficult to understand. Falls and Kelsey (1989) found contextual control of wet dog shakes only when withdrawal was precipitated by naloxone. Apparently, the effects of the morphine-associated cues and the antagonist summated to elicit this withdrawal sign. In the present experiment, presumably more physiological withdrawal was occurring in P75 than in P10, and this may have summated with the withdrawal elicited by the DE.

These results replicate and extend previous contextual withdrawal studies. Azorlosa et al. (1994) found contextual rearing, circling, and jumping $24 \mathrm{~h}$ after the final $10-\mathrm{mg} / \mathrm{kg}$ dose of morphine. Falls and Kelsey (1989) and Kelsey et al. (1990) found that paired groups showed increased rearing and circling $24 \mathrm{~h}$ after the final dose, respectively, of 30 or $50 \mathrm{mg} / \mathrm{kg}$. Falls and Kelsey also found more wet dog shakes in the paired condition, but only when withdrawal was precipitated by naltrexone.

In the present experiment, retention of rearing, darting, and jumping was found in the $10-\mathrm{mg} / \mathrm{kg}$ condition, and retention of rearing was found with $75 \mathrm{mg} / \mathrm{kg}$. Also, P75 jumped more than U75. Azorlosa et al. (1994) found retention of rearing and jumping with $10 \mathrm{mg} / \mathrm{kg}$. Kelsey et al. (1990) showed retention of rearing and circling with a terminal dose of $40 \mathrm{mg} / \mathrm{kg}$. In all these studies, with doses ranging from 10 to $75 \mathrm{mg} / \mathrm{kg}$, indices of hyperactivity were found to be the predominant contextual withdrawal signs when they were assessed with a placebo after a period of drug abstinence.

The results of this study are compatible with Siegel's $(1975,1989)$ model in which drug withdrawal is defined by the compensatory responses elicited by stimuli previously paired with morphine. According to this model, context-elicited measures of hyperactivity, such as rearing, darting, jumping, and circling, are compensatory
CRs for the sedative effects of morphine, and wet dog shakes, an index of hypothermia, are compensatory for morphine's hyperthermic effects. Although this interpretation is consistent with Siegel $(1975,1989)$, it is speculative, because the rats were not tested for sedation or hyperthermia during any of the morphine sessions. Previous research has shown that doses of 4 and $16 \mathrm{mg} / \mathrm{kg}$ of morphine produce an unconditioned decrease in activity (Mucha et al., 1981) as does $40 \mathrm{mg} / \mathrm{kg}$ (Hinson \& Siegel, 1983).

The presence of several withdrawal signs after 10 days of abstinence demonstrates that the passage of time is not sufficient to eliminate context-specific withdrawal. If context-specific withdrawal plays a role in relapse (Hinson et al., 1986; Wikler \& Pescor, 1967; but see Krank \& Perkins, 1993; Sobrero \& Bouton, 1989), it is important to determine whether it can be extinguished. Although numerous studies have demonstrated extinction of context-specific tolerance to several of morphine's effects, including analgesia (MacRae \& Siegel, 1987; Siegel, 1975, 1977; Siegel, Sherman, \& Mitchell, 1980), hyperthermia (Siegel, 1978), and sedation (Fanselow \& German, 1982), extinction of context-specific withdrawal has been investigated in only a few (O'Brien, Ehrman, \& Ternes, 1986; Schnur, 1992). In those studies, conditioned withdrawal was produced by pairing the context cues with antagonist-precipitated withdrawal. No research has examined extinction of contextual withdrawal elicited by cues paired with morphine. If tolerance and withdrawal arise from the same underlying process, techniques used to diminish context-specific tolerance should also be effective in diminishing contextspecific withdrawal.

Experiment 2 was designed to determine whether contextual withdrawal could be eliminated by extinction trials in which the contextual cues previously paired with morphine were subsequently presented without the drug. In addition, the role of injection cues in the extinction of context-specific withdrawal was examined. Several investigators have suggested that injection cues may contribute to conditioned drug effects (McLaughlin, Dewey, \& Fanselow, 1991; Siegel, 1989; Walter \& Riccio, 1983). There are at least two ways in which injection cues could become conditioned. First, the injection cues, because they are sometimes followed by morphine, may become another CS in addition to the DE. This seems unlikely, given the paired/unpaired design typically used in studies of contextual tolerance and withdrawal. In this design, injections are equally likely to be followed by morphine or saline. CSs that are relatively poor predictors of the US usually do not become well conditioned (Kamin, 1969; Rescorla, 1968). In addition, the pretraining phase in which saline injections are given might produce latent inhibition which would also retard any association between injection cues and morphine.

However, a second type of association involving the $\mathrm{DE}$ and injection cues could be formed during acquisition. The $\mathrm{DE}$, instead of functioning as a $\mathrm{CS}$, might serve 
as an occasion setter, or facilitator, which signals that the injection will be followed by the drug (Rescorla, 1985, 1986). In this analysis, the injection cues constitute the $\mathrm{CS}$, and the DE is the occasion setter which signals that the CS-US relation (injection-morphine) is in force. If this is the case, then simply placing an animal in the DE without an injection should not extinguish contextspecific withdrawal. Previous research has shown that presentations of a facilitator, without either the CS or the US, does not diminish its power (Rescorla, 1985, 1986).

In Experiment 2, four groups of rats received morphine in the DE and saline in the HC. Unpaired groups were not included in this study, because there was no evidence of any withdrawal in the unpaired condition in Experiment 1 . Although unpaired groups were not included in this study, $\mathrm{HC}$ injections of saline were given so that the "paired" treatment would be comparable to that of Experiment 1. As in Experiment 1, there were 11 acquisition sessions. Because only signs of hyperactivity were retained in Experiment 1 in both doses and more were retained in the $10-\mathrm{mg} / \mathrm{kg}$ condition, this dose was chosen for Experiment 2.

Experiment 2 incorporated two minor procedural changes. First, individual desk lamps containing $25-\mathrm{W}$ red bulbs were directed at each cage in Room 1 and the 45-W lamp was removed. Second, withdrawal testing was videotaped.

\section{EXPERIMENT 2}

\section{Method}

Subjects. Subjects were 40 male Sprague-Dawley rats weighing 197-225 $\mathrm{g}$ at the start of the experiment. They were housed as in Experiment 1.

Drugs. Morphine sulfate $(10 \mathrm{mg} / \mathrm{kg})$ and saline were administered as in Experiment 1.

Procedure. The animals underwent 3 days of pretraining, which were identical to those of Experiment 1. Following pretraining, the animals were randomly assigned to one of two conditions: Paired (morphine in the DE, saline in the $\mathrm{HC}, n=32$ ) or Sal (saline in the DE and $\mathrm{HC}, n=8$ ). As in Experiment 1, there were 11 acquisition sessions. The interdose interval was $48-72 \mathrm{~h}$ and $\mathrm{HC}$ injections were given $24 \mathrm{~h}$ later.

Twenty-four hours after the last acquisition session, withdrawal was assessed for $10 \mathrm{~min}$ following a saline injection. Rearing scores, collected from this test, were used to assign paired animals to one of four groups $(n=8)$. Rearing was chosen as the criterion because it was the most reliable and robust sign in Experiment 1 and in previous studies (Azorlosa et al., 1994; Falls \& Kelsey, 1989; Kelsey et al., 1990). As in Experiment 1, $24 \mathrm{~h}$ after the withdrawal test, the animals received one additional acquisition trial to compensate for any test effects.

Seventy-two hours after this additional session, the extinction/rest phase began. Two groups received extinction ( $E$ ) trials in the $D E$, while the other two groups rested $(R)$ in the colony room The groups that received extinction in the DE were carried to the assigned room and placed in the assigned cage. One group (EI) was injected with saline prior to placement in the box, and the other group (E) was placed in the box without an injection. The rats were left in the DE for $1 \mathrm{~h}$. During this time, the animals in the rest condition were removed from their cages and placed in individual carrying boxes in the colony room for $1 \mathrm{~h}$. This was done to equate time away from the home cages in all groups. The rats in Group RI were given an injection of saline prior to being returned to their home cages, and Group $\mathrm{R}$ was simply returned to the home cage. During the extinction/rest manipulation, half of the saline control group received placebo injections in the DE while the others were treated like Group R. There were 8 extinction trials over a period of 20 days. The extinction/rest manipulation was run at the same time of day with the same interdose interval (48-72 h) as that for the acquisition sessions. Forty-eight hours after the final extinction/rest session, all animals were transported to the DE, injected with saline, and videotaped for $10 \mathrm{~min}$. The tapes were scored by a single observer who was blind to group assignment.

\section{Results}

One animal in Group $\mathrm{R}$ was removed from the experiment because of a significant weight loss. There were no differences between the two saline groups, so their scores were combined.

Figure 4 shows the mean number of rears observed during the withdrawal retention test, conducted after the extinction/rest phase. The animals in both extinction conditions reared less than the animals in both rest conditions. There were no differences among animals in the extinction groups (E and EI) and Sal. A one-way ANOVA revealed a significant effect for groups $[F(4,34)=$ $15.75, p<.001]$, and Newman-Keuls tests confirmed the impression from Figure 4 that Groups RI and R reared more than Groups EI, E, and Sal. In addition, EI, E, and Sal were not different, nor were RI and R.

A one-way ANOVA performed on the wet dog shake scores showed no differences among the groups either before or after the extinction/rest phase. As in Experiment 1, the scores for jumps, circles, and darts (Table 2) showed marked heterogeneity of variance, so nonparametric tests were performed. There were no differences between any of the groups either before or after the extinction/rest phase (Mann-Whitney $U$ tests, one-tailed, $p<.05$ ). Wilcoxon tests (one-tailed; Siegel, 1956) examined differences between the first and second with-

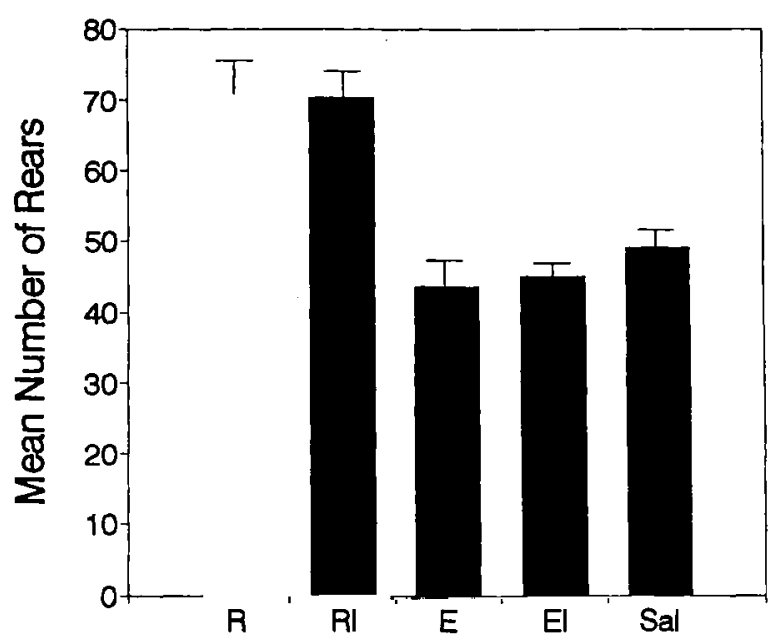

Figure 4. Mean rearing scores during the retention test in Experiment 2. 
Table 2

Median Withdrawal Scores From Experiment 2

\begin{tabular}{lccccc}
\hline $\begin{array}{l}\text { Withdrawal } \\
\text { Sign }\end{array}$ & E & EI & R & RI & Sal \\
\hline \multicolumn{7}{c}{ First } & Withdrawal & Test \\
Circles & 0.5 & 0.0 & 2.0 & 1.0 & 0.5 \\
Jumps & 3.0 & 3.5 & 2.0 & 2.0 & 4.5 \\
Darts & 0.0 & 0.0 & 0.0 & 0.0 & 0.0 \\
\multicolumn{7}{c}{} \\
Circles & Withdrawal Test After Extinction/Rest \\
Jumps & 0.0 & 0.5 & 0.0 & 2.0 & 0.5 \\
Darts & 2.0 & 1.5 & 2.0 & 0.5 & 2.0 \\
\hline
\end{tabular}

Note-E, extinction; EI, extinction, injected; R, rest; RI, rest, injected; Sal, saline.

drawal tests for each group. No group exhibited significant changes for any sign.

\section{Discussion}

Experiment 2 showed that context-specific rearing was retained for 21 days, a much longer time interval than in previous studies. It is the first to demonstrate that a withdrawal sign elicited by cues previously paired with morphine can be extinguished when the cues are repeatedly presented without the drug. Exposure to the DE, with or without a saline injection, resulted in extinction and placebo injections in the HC had no effect; Groups $\mathrm{E}$ and EI were virtually identical, as were R and RI (Figure 4). This suggests that injection cues did not become associated with the drug, probably because these cues, in comparison with the contextual cues of the DE, did not reliably predict morphine. However, it is certainly possible that injection cues could gain control over contextual withdrawal if they were always followed by the drug or if injections were given with a less salient contextual CS. Using the paired/unpaired design, Cepeda-Benito and Tiffany (1995) found that injection cues mediated associative tolerance in the unpaired groups but had no effect in the paired groups, presumably because the distinctive cues overshadowed the injection cues in the paired groups.

There was also no evidence that the DE functioned as a facilitator which signaled that the injection would be followed by morphine. If the $\mathrm{DE}$ were a facilitator, exposure to it with an injection (Group EI) would have produced more extinction than occurred in Group E, which was exposed to the DE without an injection (Rescorla, 1985, 1986). However, E and EI displayed the same low levels of rearing.

The demonstration of retention and extinction in Experiment 2 must be qualified by the fact that only contextual rearing was observed, whereas in Experiment 1, with the same dose, rearing, jumping, and darting were retained. This is similar to the results in Azorlosa et al. (1994), in which only rearing was observed in Experiment 1 but rearing, circling, and jumping were found in Experiment 2. If these measures of hyperactivity are compensatory responses, perhaps some are more easily detected or more robust (Siegel, 1989). Hinson, Poulos, and Cappell (1982) have suggested that compensatory CRs may not easily be observed in the absence of the drug but that a pharmacological challenge would result in an observable compensatory CR. This may explain why contextual control of some withdrawal signs, such as wet dog shakes, is more likely to be found when the animal is also given an opiate antagonist (Falls \& Kelsey, 1989; Kelsey et al., 1990).

\section{GENERAL DISCUSSION}

These two experiments replicated previous demonstrations that environmental cues repeatedly paired with morphine elicit withdrawal signs (Azorlosa et al., 1994; Falls \& Kelsey, 1989; Kelsey et al., 1990; Krank \& Perkins, 1993). Experiment 1 is the first in which the acquisition and retention of contextual withdrawal with a moderate $(10-\mathrm{mg} / \mathrm{kg})$ morphine dose have been compared with the acquisition and retention of contextual withdrawal with a high $(75-\mathrm{mg} / \mathrm{kg})$ morphine dose. Although somewhat different patterns of withdrawal were observed $24 \mathrm{~h}$ after the final morphine injection, the two doses produced similar patterns when subjects were tested after 10 days of drug abstinence. On the retention test, signs of hyperactivity were prominent.

Experiment 2 is the first demonstration that a contextspecific morphine withdrawal sign, elicited by cues that had been paired with the drug, can be extinguished. The finding that rearing was retained during the 3 -week extinction/rest phase of the study is consistent with the idea (Siegel, 1989) that contextual withdrawal is a CR, and as such, will persist unless extinguished. Previous studies have shown that context-specific withdrawal can be extinguished in hamsters (Schnur, 1992) and humans (O'Brien et al., 1986). However, in these studies, withdrawal was conditioned by pairing neutral cues with antagonist-precipitated withdrawal. Indeed, this type of conditioned withdrawal has been demonstrated in many studies and can be accomplished by pairing the cues with either precipitated withdrawal (e.g., O'Brien et al., 1986; Schnur, 1992; Schnur \& Wainwright, 1992) or abstinent withdrawal (e.g., Wikler \& Pescor, 1967). This type of conditioned withdrawal is difficult to accommodate within the theoretical framework of Siegel (1989) and may be more easily conditioned than the contextual withdrawal created by pairing cues with morphine (e.g., Schnur \& Wainwright, 1992).

With the exception of context-elicited wet dog shakes seen in Group P75 on the first withdrawal test of Experiment 1 , all the signs observed reflected hyperactivity. An alternative interpretation of these signs is that they represent not conditioned withdrawal, but incentive motivation. It is well established that opiates are positive reinforcers (see Young \& Herling, 1986). Stimuli repeatedly paired with a positive reinforcer of food come to elicit increases in activity (e.g., Campbell \& Sheffield, 1953). It is possible that in the present experiments, the DE elicited more rearing, jumping, and darting in the paired groups because it functioned as a conditioned positive reinforcer. 
Although this possibility cannot be ruled out, our preferred interpretation is that these behaviors reflected conditioned withdrawal. In other studies using similar doses (Azorlosa et al., 1994; Falls \& Kelsey, 1989; Kelsey et al., 1990), contextual withdrawal consisted mostly of hyperactivity in abstinent rats given saline. Kelsey and colleagues also showed contextual control of wet dog shakes, genital licking, feces excretion, and weight loss, but only when withdrawal was precipitated by naltrexone. During antagonist-precipitated withdrawal, signs of hyperactivity (e.g., rearing and circling) were much less frequent. This suggests that contextual control of withdrawal signs such as wet dog shakes, genital licking, and weight loss are more likely after precipitated withdrawal or perhaps with a much higher dose of morphine. Although contextual control of wet dog shakes was shown without an antagonist in Experiment 1, it was not retained. Wikler and Pescor (1967) found retention of wet dog shakes during a 72-day period of morphine abstinence. The terminal dose was substantially higher $(200 \mathrm{mg} / \mathrm{kg})$ than that used in other studies of contextual withdrawal, and conditioning was obtained by pairing the context with withdrawal rather than morphine. Perhaps wet dog shakes, even after an extended period of drug abstinence, could be elicited by cues previously paired with a much higher dose of morphine than that used in the present studies. As far as we can determine, the $75-\mathrm{mg} / \mathrm{kg}$ dose used in Experiment 1 is the highest dose used to study contextual withdrawal elicited by cues paired with morphine.

The issue of whether cue-elicited rearing, darting, and jumping found in the present experiments and in previous research (Azorlosa et al., 1994; Falls \& Kelsey, 1989; Kelsey et al., 1990) indicate withdrawal or a positive incentive state is an important theoretical question. Unfortunately, the present experiments do not allow for a definitive choice between these alternatives. However, these experiments do show that context-elicited hyperactivity persisted for a long period of drug abstinence unless it was systematically extinguished. According to Siegel (1989) these contextual cues may lead to relapse even after a prolonged period of abstinence. Hinson et al. (1986) showed that morphine-abstinent rats drank more of a morphine solution in the presence of cues previously paired with morphine injections than rats who had those cues paired with saline. However, two later studies failed to find this effect (Krank \& Perkins, 1993; Sobrero \& Bouton, 1989). Clearly, additional research is needed to determine what motivational state is elicited by cues paired with an opiate, whether these cues will motivate self-administration after a long period of abstinence, and if so, whether an extinction procedure reduces self-administration.

\section{REFERENCES}

Azorlosa, J. L., Hartley, N. E., \& Deffner-Rappold, C. (1994). Context-specific morphine tolerance and withdrawal: The effects of interdose interval. Psychobiology, 22, 304-311.

Blasig, J., Herz, A., Reinhold, K., \& Zieglgänsberger, S. (1973). Development of physical dependence on morphine in respect to time and dosage of quantification of the precipitated withdrawal syndrome in rats. Psychopharmacologia, 33, 19-38.

Campbell, B. A., \& Sheffield, F. D. (1953). Relation of random activity to food deprivation. Journal of Comparative \& Physiological Psychology, 46, 320-322.

Cepeda-Benito, A., \& Tiffany, S. T. (1995). Role of drug-administration cues in the associative control of morphine tolerance in the rat. Psychopharmacology, 122, 312-316.

Chorot, P., \& SANDíN, B. (1993). Effects of US intensity and duration of exposure of nonreinforced $\mathrm{CS}$ on conditioned electrodermal responses: An experimental analysis of the incubation theory of anxiety. Psychological Reports, 73, 931-941.

EYSENCK, H. J. (1979). The conditioning model of neurosis. Behavioral \& Brain Sciences, 2, 155-199.

Falls, W. A., \& Kelsey, J. E. (1989). Procedures that produce context-specific tolerance to morphine in rats also produce contextspecific withdrawal. Behavioral Neuroscience, 103, 842-849.

Fanselow, M. S., \& German, C. (1982). Explicitly unpaired delivery of morphine and the test situation: Extinction and retardation of tolerance if the suppressing effects of morphine on locomotor activity. Behavioral \& Neural Biology, 35, 231-241.

Goudie, A. J., \& DemellweEk, C. (1986). Conditioning factors in drug tolerance. In S. R. Goldberg \& I. P. Stolerman (Eds.), Behavioral analysis of drug dependence (pp. 225-285). New York: Academic Press.

Hinson, R. E., Poulos, C. X., \& Cappell, H. (1982). Effects of pentobarbital and cocaine in rats expecting pentobarbital. Pharmacology, Biochemistry \& Behavior, 16, 661-666.

Hinson, R. E., Poulos, C. X., Thomas, W., \& Cappell, H. (1986). Pavlovian conditioning and addictive behavior: Relapse to oral selfadministration of morphine. Behavioral Neuroscience, 100, 368-375.

Hinson, R. W., \& SiEgEL, S. (1983). Anticipatory hyperexcitability and tolerance to the narcotizing effect of morphine in the rat. $B e$ havioral Neuroscience, 97, 759-767.

KaLOUPEK, D. (1983). The effects of compound in vivo and imaginal exposure: A test of fear enhancement models. Behavior Therapy, 14, 345-356.

KAMIN, L. J. (1969). Predictability, surprise, attention, and conditioning. In B. A. Campbell \& R. M. Church (Eds.), Punishment and aversive behavior (pp. 279-296). New York: Appleton-CenturyCrofts.

Kelsey, J., Aranow, J., \& Matthews, R. (1990). Context-specific morphine withdrawal in rats: Duration and effects of clonidine. $B e$ havioral Neuroscience, 104, 704-709.

Krank, M. D., \& Perkins, W. L. (1993). Conditioned withdrawal signs elicited by contextual cues for morphine administration. Psychobiology, 21, 113-119.

LiNESMAN, M. A. (1977). Naloxone-precipitated withdrawal as a function of the morphine-naloxone interval. Psychopharmacologia, 54, $159-164$

MacRae, J. R., \& Siegel, S. (1987). Extinction of tolerance to the analgesic effect of morphine: Intracerebroventricular administration and the effects of stress. Behavioral Neuroscience, 101, 790-796.

Mclaughlin, C. R., Dewey, W. L., \& Fanselow, M. S. (1991). Shortand long-term factors in tolerance to morphine-induced antinociception: Single or multiple mechanisms? Psychobiology, 19, 217-222.

Mucha, R. F., Volkovskis, C., \& Kalant, H. (1981). Conditioned increases in locomotor activity produced with morphine as an unconditioned stimulus, and the relation of conditioning to acute morphine effect and tolerance. Journal of Comparative \& Physiological Psychology, 95, 351-362.

NicholaichuK, T. P., QueSNEL, L. J., \& TAIT, R. W. (1982). Eysenck's theory of incubation: An empirical test. Behavior Research \& Therapy, 20, 329-338

O'Brien, C. P., Ehrman, R. N., \& Ternes, J. W. (1986). Classical conditioning in human opioid dependence. In S. R. Goldberg \& I. P. Stolerman (Eds.), Behavioral analysis of drug dependence (pp. 329356). Orlando, FL: Academic Press.

Rescorla, R. A. (1968). Probability of shock in the presence and absence of $\mathrm{CS}$ in fear conditioning. Journal of Comparative \& Physiological Psychology, 66, 1-5.

RESCORLA, R. A. (1985). Conditioned inhibition and facilitation. In 
R. R. Miller \& N. E. Spear (Eds.), Information processing in animals: Conditioned inhibition (pp. 299-326). Hillsdale, NJ: Erlbaum.

RESCORLA, R. A. (1986). Extinction of facilitation. Journal of Experimental Psychology: Animal Behavior Processes, 12, 16-24.

Rohrbaugh, M., \& Riccio, D. C. (1970). Paradoxical enhancement of learned fear. Journal of Abnormal Psychology, 75, 210-216.

Rohrbaugh, M., Riccio, D. C., \& Arthur, A. (1972). Paradoxical enhancement of conditioned suppression. Behavioral Research \& Therapy, 10, 125-130.

SCHNUR, P. (1992). Conditioned morphine withdrawal in the hamster. Psychopharmacology, 107, 517-522.

Schnur, P., \& WaINWRight, M. (1992). Conditioned withdrawal in environments associated with the presence or absence of morphine. Pharmacology, Biochemistry \& Behavior, 41, 543-546.

SIEGEL, S. (1956). Nonparametric statistics for the behavioral sciences. New York: McGraw-Hill.

SIEGEL, S. (1975). Evidence from rats that morphine tolerance is a learned response. Journal of Comparative \& Physiological Psychology, 89, 498-506.

SiEgEL, S. (1977). Morphine tolerance as an associative process. Journal of Experimental Psychology: Animal Behavior Processes, 3 , $1-13$.

SIEGEL, S. (1978). Tolerance to the hyperthermic effect of morphine in the rat is a learned response. Journal of Comparative \& Physiological Psychology, 92, 1137-1149.
SIEGEL, S. (1989). Pharmacological conditioning and drug effects. In A. J. Goudie \& M. W. Emmett-Oglesby (Eds.), Psychoactive drugs (pp. 115-180). Clifton, NJ: Humana Press.

Siegel, S., Sherman, J. E., \& Mitchell, D. (1980). Extinction of morphine analgesic tolerance. Learning \& Motivation, 11, 289-301.

Sobrero, A. P., \& Bouton, M. E. (1989). Effects of stimuli present during oral morphine administration on withdrawal and subsequent consumption. Psychobiology, 17, 179-190.

WALTER, T. A., \& RICCIO, D. C. (1983). Overshadowing effects in the stimulus control of morphine analgesic tolerance. Behavioral Neuroscience, 97, 658-662.

WIKLER, A., \& PESCOR, F. T. (1967). Classical conditioning of a morphine abstinence phenomenon, reinforcement of opioid-drinking behavior and "relapse" in morphine-addicted rats. Psychopharmacologia, 10, 255-284.

Young, A. M., \& HerLing, S. (1986). Drugs as reinforcers: Studies in laboratory animals. In S. R. Goldberg \& I. P. Stolerman (Eds.), Behavioral analysis of drug dependence (pp. 9-67). New York: Academic Press.

(Manuscript received August 7, 1995; revision accepted for publication June 25,1996 .) 